\title{
Los tratados silogísticos de Boecio: sus fuentes, historia e influencia en la cristiandad latina
}

\section{DESCRIPCIÓN Y FUENTES DEL TRATADO}

El De syllogismo categorico (=DSC) es un tratado sobre lógica aristotélica escrito por Boecio el 505 o 506 de nuestra era (1). A pesar de la importancia que este tratado tiene para comprender no solo la historia del proceso de transmisión de la lógica de Aristóteles al medioevo latino, sino también la correcta interpretación de la doctrina lógica en la antigüedad, el texto no ha recibido ni una edición crítica ni un examen filológico completo (2).

El DSC se encuentra en los códices más antiguos (por ejemplo, Orleáns 267, p. 57) con el título "Introductio in cathegoricos syllogi" (estas faltas de ortografía son comunes en los códices más antiguos), y en la revisión que hicieron Martianus Rota (Venecia 1543) y Henrichus Loritus Glareanus (Basilea 1546) su título ya aparece cambiado (no corrupto) a De syllogismo categorico. Nuestra edición moderna (Migne 1891) está basada en estas dos ediciones del siglo XVI, razón por la cual el texto se encuentra titulado como De syllogismo categorico. Durante el siglo XX, la mayoría de los especialistas han corregido este título por el de De syllogismis categoricis, después de que Brandt (1903), p. 238, n. 4, argumentara a favor del uso del plural, atendiendo a que Boecio se refiere a él como una introducción a los silogismos categóricos.

En este tratado no solo confluye la enseñanza de Aristóteles, sino también la del neoplatónico Porfirio y de los peripatéticos Teofrasto y Eudemo, asociados de Aristóteles en el Liceo. En cuanto a sus fuentes, Boecio en DSC está siguiendo cierto material basado en una Introducción a los Silogismos Categóricos que Porfirio escribió para evaluar y aprobar la teoría silogística de Teofrasto y Eudemo. Esto se desprende directamente de lo que Boecio dice al final de esta obra (DSC 829D5-830D12):

(1) Para la cronología: De Rijk (1964).

(2) Con todo, a la fecha Christina Thörnqvist (2006) trabaja actualmente en su edición en la Universidad de Gotemburgo. 
"Y estas cosas <expuestas> las tomé de una introducción sobre los silogismos categóricos, siguiendo a Aristóteles mayormente, pero pidiendo prestado (mutuatus) algunas cosas a Teofrasto y Porfirio, tanto cuanto lo permite la brevedad <que hay que mantener $>$ al introducir <un asunto $>$ ".

Así, las fuentes de DSC son un material basado en la exposición que Porfirio hizo en el IV d. C. sobre la teoría lógica de Teofrasto, teoría de la cual tenemos noticias a través de varias obras cuyos títulos se encuentran citados por distintos autores antiguos, por ejemplo, Sobre la afirmación y la negación, citado por Boecio (3). Los Primeros Analíticos, citado por Alejandro de Afrodisia (4), etc. (5). Por su parte, la existencia de una obra titulada 'Introducción a los silogismos categóricos' escrita por Porfirio, queda corroborada por las investigaciones del especialista en la vida y obras de Porfirio J. Bidez (6).

Pero que sea un material postporfiriano el que adopta Boecio en su DSC no impide que posea enseñanzas postaristotélicas de raigambre peripatética. Esto parece haber quedado bien establecido por RP. Isaak (1953), p. 122, al mostrar que DSC tiene una fuente común con un texto de lógica del siglo II d. C., es decir, unos 100 años antes que Porfirio, titulado Peri Hermeneias y escrito por el platónico Apuleio de Madaura. Esta opinión se ve confirmada por una comparación de los dos textos hecha por Sullivan (1967), 210-228, aunque la hipótesis de Sullivan (1967), p. 226, de que Boecio pudo haber tenido a su disposición una copia del Peri Hermeneias de Apuleio y haber sufrido influencia de Apuleio, no ha recibido mayor confirmación.

\section{CONTENIDO}

El contenido de este tratado es una exposición sobre lógica aristotélica, es decir, sobre proposiciones y silogismos categóricos y sus elementos. El texto de DSC se encuentra dividido en dos libros o secciones. En el primero, Boecio hace una revisión de la teoría de las proposiciones, de un modo muy completo, y con bastante similitud al modo como trata este tema en su comentario al De Interpretatione de Aristóteles (=in Int), editado por Meiser (1877-1880). Sin embargo, en el primer libro de DSC, la enseñanza de Boecio sobrepasa notablemente lo que Aristóteles enseña en De Interpretatione al (i) incluir la relación de subalternación cuando revisa las relaciones lógicas del cuadrado de las oposiciones; y (ii) hacer una amplia exposición sobre conversión, incluyendo la conversión por contraposición (que Aristóteles solo trata para la afirmativa universal); y (iii) generalizar los resultados sobre la conversión por accidente, ampliándola a la negativa particular (cosa que Aristóteles no hace). Boecio entrega las respectivas demostraciones lógicas de cada una de las nuevas operaciones definidas, haciendo una exposición muy original y coherente desde un punto de vista lógico.

(3) Cf. in Int 2, 9, 25 (= Meiser 1887-1880).

(4) in An $\operatorname{Pr} 123,19 ; 388,18$ (=Wallies 1883).

(5) Sobre estos títulos, ver Sharples (1992), pp. 114-123.

(6) Bidez (1923), p. 198 y Bidez (1964), p. 66*. 
En el segundo libro aparece la teoría del silogismo y, nuevamente, no son solo Aristóteles y sus Primeros Analíticos acogidos, sino también Teofrasto y la discusión de Porfirio sobre ella. A los cuatro modos válidos de la primera figura distinguidos por Aristóteles (y reconocidos más tarde por los nombres Barbara, Celarent, Darii y Ferio) se suman los cinco descubiertos por Teofrasto en esta misma figura, que luego el siglo XIV los conoció como los modos de la primera figura indirecta: Baralipton, Celantes, Dabitis, Fapesmo y Frisesomorum. El segundo libro de DSC termina con una explicación de la definición del silogismo muy similar a la exposición que Alejandro de Afrodisia hace de ella en su comentario a los Tópicos de Aristóteles y con una explicación de la doctrina de la restitución de los modos imperfectos de la segunda y tercera figuras silogísticas a los cuatro primeros modos de la primera figura, que es más técnica y elaborada que la que Aristóteles presenta en Analíticos Primeros.

La consideración atenta de los contenidos lógicos de DSC hace manifiesto que Boecio sigue una división de las proposiciones categóricas que tiene la siguiente característica: desde ella es posible generar las principales operaciones lógicas definidas en la lógica aristotélica: la oposición de las proposiciones (la contradicción, la contrariedad y la subcontrariedad); la conversión (simple, por accidente y por contraposición); y los silogismos, con sus principales extensiones (7). En efecto, luego de comentar sobre el nombre, el verbo, los nombres y verbos indefinidos, los casos del nombre y del verbo; sobre la frase u oración y, finalmente, sobre la proposición, Boecio divide las proposiciones en aquellas cuyos términos (a) nada tienen en común entre sí de aquellas que (b) algo tienen en común entre sí. Ejemplos de (a) son 'todo hombre es animal' y 'la virtud es buena', pues estas dos proposiciones no tienen ningún término en común. En cuanto a (b), las proposiciones que tienen algún término en común pueden o bien (b.1) tener ambos términos en común o bien (b.2) tener algún término en común. En (b.1) encontramos dos tipos: (b.1.1) según el mismo orden y (b.1.2) según un cambio de orden. Ejemplos de (b.1.1) son: 'todo hombre es animal' y 'ningún hombre es animal', pues estas dos proposiciones tienen el sujeto y el predicado en común (es decir, ambos términos) y su orden no varía. Pero como varía la cantidad o la calidad de las proposiciones, la teoría semántica del cuadrado de las oposiciones y la teoría de la conversión se generan y operan con este tipo de proposiciones. Por su parte, ejemplos de (b.1.2) son: 'todo hombre es animal', 'todo animal es hombre', pues estas proposiciones tienen los dos términos en común, pero no conservan el mismo orden. Este es el tipo de proposición que estudia la teoría de la conversión. En cuanto a (b.2), o sea, proposiciones que tienen algún término en

(7) Aunque no lo discutiré aquí, mi tesis de que esta división genera las principales operaciones lógicas definidas por Aristóteles no es advertida por De Rijk (1964), p. 18. Según establece este erudito holandés en su muy completo estudio sobre la cronología de los escritos de Boecio, la primera rama de esta división genera la teoría de la conversión, de modo que en este punto concordamos con él. No obstante, De Rijk (ibid) sostiene que la segunda rama de la división no fue desarrollada por Boecio en este tratado ni en ninguna otra parte. Mi propia opinión es que esta opinión de De Rijk elimina, erróneamente, un argumento excelente para probar la unidad del DSC en dos libros, a saber, que hay una simetría entre la división y los tipos de operaciones definidas por Aristóteles, de modo que todas las operaciones (incluida el silogismo) están retratadas en esta división. 
común, hay tres especies: (b.2.1) aquellas que tienen el término común en el sujeto (la primera) y en el predicado (la segunda); y (b.2.2) aquellas que lo tienen en el predicado en ambas; y (b.2.3) aquellas que lo tienen en el sujeto en ambas. Desde estas tres últimas especies surgen las tres figuras silogísticas, como se ve en los siguientes ejemplos correspondientes: 'todo hombre es mortal' y 'todo griego es hombre' $\left(=1^{\mathrm{a}}\right)$; 'todo hombre es mortal' y 'todo animal es mortal' $\left(=2^{\mathrm{a}}\right)$; 'todo hombre es animal' y 'todo hombre es griego' $\left(=3^{\mathrm{a}}\right)$. Boecio en su comentario al De Interpretatione de Aristóteles introduce una división de la proposiciones que tiene por fin identificar todos los tipos sintácticos de proposiciones categóricas que Aristóteles distinguiría (8), pero la división de DSC no tiene esta misma intención, sino la de derivar las operaciones lógicas según las proposiciones tengan o no términos comunes. Esta división que menciono puede diagramarse de la siguiente manera:

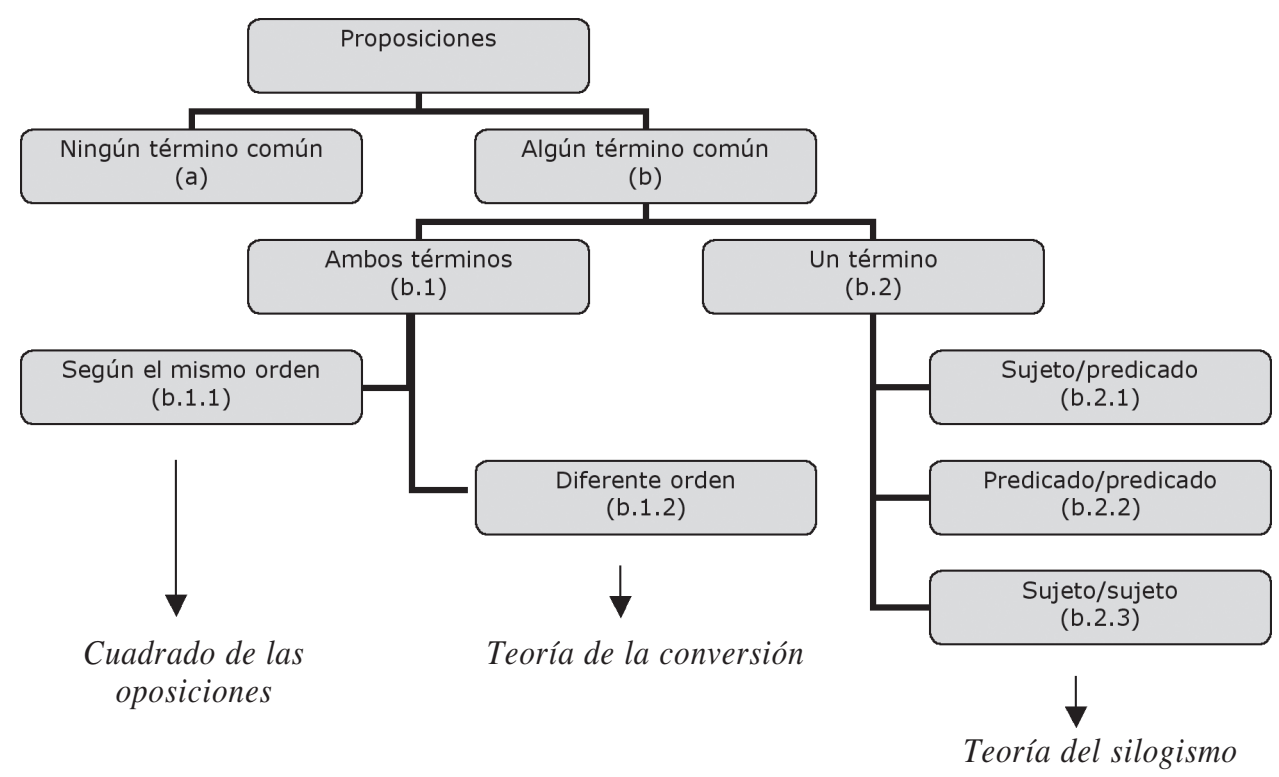

La división mencionada da coherencia argumentativa y, por tanto, unidad a DSC, pues el segundo libro de este tratado, que expone la teoría silogística con las extensiones de Teofrasto, se puede interpretar como el desarrollo de la última parte de la división, esa correspondiente a (b.2). De este modo, DSC adquiere unidad expositiva y todos los elementos teóricos de que trata se ven integrados en un plan o programa expositivo que surge desde esta división.

(8) Boecio distingue 144 especies proposición en la segunda edición de su in Int $(321,20-324,24)$, (=Meiser 1877-1880). 


\section{LA INTERPRETACIÓN DE McKINLAY}

Esta tesis de la unidad de DSC se opone directamente a la interpretación que el especialista McKinlay (1907) y (1938) hizo sobre este tratado, a saber, que el primer libro de DSC es espurio y el segundo libro de DSC (sobre silogística) es en realidad el segundo libro de otro tratado de lógica escrito por Boecio, emparentado y muy similar al anterior, conocido actualmente por el título Introductio ad syllogismos categoricos (=ISC). También editado en Migne (1891).

MacKinlay, en efecto, sabía que en los códices más antiguos (9), el DSC se acompaña de otro tratado sobre lógica aristotélica titulado allí como Liber anti praedicamenta, conocido posteriormente en las ediciones del XVI como Introductio ad syllogismos categoricos (=ISC). Su similitud con DSC es enorme y ello hizo creer a Prantl (1855), I, p. 682, n. 80, que el primer libro de DSC era un extracto (excerpt) del primer y único libro de ISC, pero Brandt (1903), p. 245, mostró la debilidad de la hipótesis, simplemente mostrando que en DSC no están presentes algunas piezas de la teoría de ISC, en particular las proposiciones con términos indefinidos. Por este tiempo, un autor italiano, Rocco Murari, publicaba su Dante e Boezio (1905), donde se destacaban las peculiaridades estilísticas del DSC. Murari (1905), p. 92, fue el primero en sostener que el primer libro de DSC no era de Boecio. Murari creyó que era un resumen medieval, muy posterior al tiempo de Boecio. Fue entonces cuando McKinlay decidió aplicar el método estilométrico a toda la obra de Boecio con el fin de que este método (que había sido aplicado con gran éxito por Lutoslawski en 1897 a las obras de Platón) pudiera entregar nuevos datos sobre la cronología de las obras de Boecio y resolver el problema propuesto por Murari (1905).

Los resultados estilométricos de McKinlay (1907) fueron sorprendentes: el DSC presentaba serias complicaciones estilométricas, especialmente el libro primero, tanto así que McKinlay lo llamaba un texto 'peculiar' (1907, p. 140) y 'abnormal' (1907, p. 144). McKinlay dudaba de la tesis de Murari, pero los resultados estilométricos confirmaban la hipótesis del italiano. Entonces, después de 30 años, McKinlay en su nueva publicación de 1938 refrendó la hipótesis de Murari y agregó, para darle mayor solidez a ella, que los títulos de los tratados estaban cambiados ya en los códices más antiguos: que el título de Introductio ad syllogismos categoricos debería ser dado a la unión del segundo libro de nuestro actual DSC con el ISC de nuestros códices más modernos. En otras palabras, de los dos tratados sobrevivientes debería hacerse solo uno, despreciando el libro primero de DSC.

Aunque los títulos de DSC e ISC en los códices más antiguos sean efectivamente otros -hecho que da cierta fuerza a la hipótesis de McKinlay-, lo cierto es que De Rijk (1964), p. 39, junto con Magee (1998), XVII-XIX, han expresado desconfianza sobre la utilización del método estilométrico hecho por McKinlay, sobre la base de la no existencia de una edición crítica de los tratados (10). En efecto, la edición de Migne (1891) es moderna, pero no una edición crítica.

(9) Sobre la historia de los manuscritos de los tratados lógicos mencionados la mejor información se halla en Van de Vyver (1929), pp. 425-452. También Magee (1998), LIX-LXXV. Y también De Rijk (1964), pp. 5-6.

(10) En una nota privada, muy recientemente, la profesora Thörnqvist me manifiesta que es de la misma opinión. 


\section{LA INFLUENCIA DE LOS TRATADOS}

Los tratados mencionados nunca fueron considerados espurios o asociados a alguna tradición que dudara de la autoría de Boecio, y no obstante no hay signos de que hayan sido acogidos por los lógicos y filósofos medievales antes del siglo XI. Son pues cinco siglos donde las referencias a estos tratados son nulas. Una explicación de esta singularidad puede ser el que la mayor tradición que generan los comentarios a las Categorías, al De Interpretatione y al Isagoge de Porfirio, textos que componían la enseñanza de la así llamada logica vetus, haya eclipsado la enseñanza de estos tratados boecianos. Pero como en DSC e ISC hay una revisión actualizada de todos los aspectos más útiles de la lógica aristotélica, hecha con mucha claridad y concisión, que los primeros medievales habrían acogido con la mayor complacencia, es plausible que los tratados referidos no hayan ejercido una influencia directa en la enseñanza del trivium en la escuela medieval a causa de que no hayan sido conocidos por los primeros medievales. Una prueba de ello puede ser el conocimiento tardío de la silogística por los medievales. Es notable que tampoco el Peri Hermeneias de Apuleio, donde hay noticias y un breve tratamiento de la silogística y sus extensiones por Teofrasto, ejerció esta influencia. Es así dable aventurar la hipótesis de que de haber estado a disposición de los primeros medievales no habría habido en nuestra historia la distinción entre logica vetus y logica nova, o al menos la aparición del silogismo en la literatura filosófica y lógica no hubiera marcado la diferencia entre estas dos lógicas, sino solo la teoría de la demostración científica de los Analíticos Posteriores.

El primer texto de nuestra historia occidental donde se ve la influencia de su enseñanza es el Anónimo Abbreviatio Montana. El otro es la Dialectica de Pedro Abelardo. Sabemos de esta influencia no solo por el nombre de Boecio en estas obras, sino también porque las división de las proposiciones que hemos visto arriba son adoptadas totalmente en estos tratados posteriores. Otras piezas de la teoría de los tratados boecianos son también adoptadas por la Abbreviatio y por la Dialectica de Abelardo, por ejemplo, los modos indirectos de la primera figura y la conversión de la A y la O por contraposición. Esta última doctrina se debe seguramente a Porfirio, ya que Alejandro de Afrodisia en su in Top no conoce la contraposición de la $\mathrm{O}$ y no menciona la imposibilidad de contraponer válidamente la I y la $\mathrm{E}$, cosa que sí hace Boecio. En efecto, esto sugiere que la fuente de esta doctrina se hallaba en Porfirio y no en Teofrasto, porque de haberla conocido Teofrasto, seguramente también Alejandro.

Desde el siglo XI en adelante la influencia de estos tratados se hace más patente en nuestra historia, así en el siglo XIV, en la obra de Pedro Hispano, las Summulae Logicales, ya se aprecia con claridad esta influencia. Ella se aprecia en la adopción de los modos indirectos de la primera figura, en la acogida que recibe la doctrina de la materia de la proposición -que no obstante se puede rastrear en la historia de la lógica tan atrás como Alejandro de Afrodisia y el mismo Apuleio-. También estos tratados son importantes por plantear la idea de que los primeros cuatro modos de la primera figura son perfectos y funcionan, por decirlo así, como axiomas de la teoría silogística, ya que todos los otros modos son derivados y se pueden reducir y derivar de estos cuatro primeros modos canónicos. 
A pesar de toda esta influencia, los tratados podrían todavía ejercer una mayor influencia no solo cultural, por ser los primeros tratados de lógica aristotélica que la tradición cristiana conoció, verdaderos compendios de lógica aristotélica, sino por su enorme originalidad en el tratamiento de los temas lógicos tradicionales. De aquí que en cierto sentido urja una edición crítica de ellos, traducciones, comentarios y estudios.

En este sentido, me parece que es conveniente refutar definitivamente la hipótesis de Murari y McKinlay, todavía muy dependiente de la estilometría, y poco de las consideraciones doctrinales de los tratados. Para ello es importante que los estudios e investigaciones se concentren, en especial, en la comprensión de las doctrinas lógicas de estos tratados, en particular con vistas a entender el rol que juegan en estos tratados las divisiones de las proposiciones categóricas en los desarrollos de las teorías lógicas. La importancia de este esfuerzo radica en el hecho de que (i) la división de DSC es central a la exposición de los contenidos lógicos de este tratado; y (ii) la división de ISC (769B14 y ss.) es distinta a la de DSC, ya que la rama de (b.2) no se abre en ISC, pero en cambio se abre para (b.1) los correspondientes sujetos y predicados indefinidos (e.g. 'no-hombre', 'no-justo'), surgiendo así las proposiciones con términos indefinidos y las operaciones de oposición y conversión con proposiciones indefinidas, es decir, esas que tienen ya en su sujeto, ya en su predicado, ya en ambos, términos indefinidos.

Mi conjetura es que por medio de esta reorientación de los estudios e investigaciones sería posible responder algunas interrogantes que aún persisten en torno a la naturaleza y contenido de los tratados silogísticos de Boecio. Por ejemplo, ¿qué significa la ausencia de los términos indefinidos en las proposiciones de DSC? Y ¿qué significa que en ISC no se mencionen los silogismos? ¿Se vincula la división de proposiciones de ISC a las operaciones lógicas que pueden generar, tal como ocurre en DSC? ¿Qué relación hay entre los actuales contenidos de los tratados y los diversos títulos que han recibido en la tradición medieval de manuscritos? ¿Cuáles diferencias estrictas o lógicas presenta las exposiciones de DSC y la de ISC? ¿Qué es cierto y qué errado en la hipótesis de McKinlay? ¿Hay un segundo libro de ISC? ¿Debería un segundo libro de ISC tratar de silogismos con proposiciones indefinidas?

\section{LAS PRÓXIMAS INVESTIGACIONES}

Si la división de DSC resulta ser, como creo, central en la explicación del contenido y los límites de la teoría de este tratado, entonces es esperable que la división de ISC sea igualmente útil en la explicación del contenido de ISC y de sus límites teóricos. Así, podríamos acercarnos en nuestro siglo a algunos resultados que creo se podrían predecir: (a) la definitiva refutación de la hipótesis MurariMcKinlay; (b) el establecimiento de una diferencia doctrinal precisa entre los tratados, basada en los límites que las divisiones imponen a la teoría lógica de cada uno de ellos; (c) la prueba de que los dos libros de DSC son auténticos e ISC es un tratado cuyo objeto temático es distinto al de DSC, aunque complementario por incluir los términos indefinidos en los sujetos y/o los predicados de las proposiciones que están sujetas a operaciones lógicas. 
Una investigación como la que describo aquí resulta indispensable para lograr un avance respecto del estado actual del conocimiento que se tiene de estos dos tratados y de su relación (asunto, en verdad, largamente debido, y cuya carencia se muestra por: (i) la falta de ediciones críticas de estos dos tratados mencionados; (ii) la falta de traducciones a lengua moderna; y finalmente, por (iii) la falta de artículos científicos sobre la naturaleza de estos tratados y su relación). En este sentido, es importante notar que si la división de ISC es tan central al desarrollo de la doctrina de este tratado como lo es la división de DSC, entonces se podrá probar que los contenidos y límites teóricos de cada tratado son independientes, y este hecho permitirá que sus contenidos lógicos puedan ser estudiados con mayor provecho, sin necesidad de reducir uno a otro (11).

Finalmente, hay que notar que una investigación como la que aquí se describe debería aportar nuevos elementos de juicio para evaluar si la hipótesis de Isaak (1953), p. 122 (a saber, que DSC y el Peri Hermeneias del platónico Apuleio de Madaura, del siglo II d. C. tienen una fuente común), se aplica también correctamente a ISC. Por tanto, la investigación también permitiría saber si los dos tratados de Boecio, apellidados 'silogísticos', es decir, DSC e ISC, tienen una fuente común con el Peri Hermeneias de Apuleio (12).

\section{RESUMEN}

En este artículo se describen los contenidos de un tratado sobre lógica aristotélica escrito por Boecio en el VI d. C., llamado por nuestras ediciones modernas De syllogismo categorico $(=D S C)$. A través de sus contenidos, es posible mostrar: (i) la importancia de este tratado en el proceso de transmisión de la lógica de Aristóteles al medioevo latino; (ii) su relación con otro tratado monográfico similar, titulado por la crítica moderna Introductio ad syllogismos categoricos (ISC) y su relación temática con DSC; (iii) las fuentes más probables de ambos tratados; $y$, finalmente, (iv) su influencia en el medioevo latino. La hipótesis que se defiende en este artículo es que los contenidos lógicos de este tratado, en particular la clasificación de las proposiciones categóricas, contenida en el corazón del DSC, entregan no solo el objeto temático al tratado, sino también coherencia argumentativa y unidad. En especial, el estudio de esta clasificación permite demostrar la unidad de DSC y en particular que el segundo libro de DSC es genuino y no espurio como sostuvieron, no hace mucho, los estudiosos Murari y McKinlay; además, esta misma clasificación permite apreciar su diferencia con ISC, por el hecho de que este último tratado posee una clasificación diferente a la de $D S C$. La base de la hipótesis propuesta se halla en que la clasificación mencionada es un intento por generar, a partir de los elementos proposicionales, las principales operaciones lógicas definidas por Aristóteles en sus escritos lógicos, asunto que en la revisión de De Rijk (1964) no quedó totalmente establecido.

(11) Cabe destacar que Prior (1953) y (1962) realizó un estudio lógico de ISC, enriqueciendo notablemente la teoría lógica aristotélica con la lógica de las proposiciones indefinidas. Prior, sin embargo, se sintió atraído por las doctrinas lógicas de este tratado y su intención fue simplemente estudiarlas desde un punto de vista lógico (o sea, su consistencia interna) con total independencia de las cuestiones relativas a la relación de ISC con DSC que hemos mencionado aquí.

(12) Este trabajo fue posible gracias a Fondecyt Regular $N^{\circ} 1050879$ ("Formalidad y formalismo en el origen del pensamiento lógico: el caso del De syllogismo categorico de Boecio"). 


\section{ABSTRACT}

In this article, the author describes the contents of a treatise on Aristotelian logic, written by Boethius in the sixth century A.D., titled in our modern editions as De syllogismo categorico $(D S C)$. It is possible to show, through the contents: (i) the importance of this treatise for the transmission process of Aristotle's logic to the Latin medieval period; (ii) its relation to another, similar monographic treatise, titled by modern criticism as Introductio ad syllogismos categoricos (ISC) and its thematic relation to DSC; (iii) the most probable sources of both treaties; and, finally (iv), its influence upon the Latin medieval period. The hypothesis that the present article defends is that the logical contents of this treatise, in particular the classification of the categorical propositions, contained in the heart of the DSC, give not only the thematic object to the treatise, but also argumentative coherence and unity. The study of this classification, especially, permits one to demonstrate the unity of $D S C$ and, in particular, that the second book of DSC is genuine-not spurious as was claimed, not long ago, by the investigators Murari and McKinlay. In addition, this same classification allows one to appreciate its difference from ISC, by the fact that this latter treatise possesses a classification that is different from that of DSC. The basis of the proposed hypothesis is found in that the aforementioned classification is an attempt to generate, from the propositional elements, the principal logical operations defined by Aristotle in his writings on logic, a matter that, in De Rijk's revision (1964), was not entirely established.

\section{REFERENCIAS}

Bidez (1923): Bidez, J., "Boece et Porphyre", en Revue belge de philology et d'histoire, 1923, pp. 189-201.

Bidez (1964): Bidez, J., Vie de Porphyre. Le Philosophe néoplatonicien, Hildesheim 1964: G. Olms.

Brandt (1903): "Entstehungszeit und zeitliche Folge der Werke von Boethius", en Philologus, 62, 1903, pp. 234-275.

Correia (2006), Correia, M., "The Nature, Structure and Sources of Boethius' De syllogismis categoricis", artículo en redacción (Fondecyt Regular 1050879).

De Rijk (1964): De Rijk, L., On the Chronology of Boethius' works on Logic (I and II), en Vivarium, vol. 2, parts $1 \& 2,1964$, pp. 1-49 and 122-162.

Isaak (1953): Isaak, I., RP., Le Peri hermeneias en Occident de Boèce à Saint Thomas. Histoire littéraire d'un traité d'Aristote, París 1953 : Vrin.

Magee (1998): Magee, J., Anicii Manlii Severini Boethii De divisione liber. Critical edition, translation, prolegomena and commentary. Leiden/Boston/Koln: Brill.

McKinlay (1907), A. P., "Stylistic tests and the chronology of the works of Boethius, en Harvard Studies in Classical Philology, XVIII (1907), pp. 123-156.

McKinlay (1938): "The De syllogismis categoricis and Introductio ad syllogismos categoricos of Boethius", en Classical and Mediaeval Studies in honor of E. K. Rand, New York 1938, pp. 209-219.

Migne (1891): De Syllogismo Categorico, en Patrologia Latina, 64, vol. 2, J.-P. Migne (Ed.), Paris 1891. (=DSC)

Migne (1891): Introductio ad Syllogismos Categoricos, en Patrologia Latina, 64, vol. 2, J.-P. Migne (Ed.), Paris 1891. (=ISC).

Murari (1905): Murari, R., Dante e Boezio, Bologna 1905.

Prantl (1855): Prantl, C., Geschichte der Logik im Abendlande, Leipzig 1855-1870. 
Prior (1953): Prior, A.N., The Logic of the Negative Terms in Boethius, en Franciscan Studies, 13 (1953), vol. I, pp. 1-6.

Prior (1962): Prior, A.N., Formal Logic, Oxford 1962.

Sharples (1992): Theophrastus of Eresus. Sources for his Life, Writings, Thought and Influence, vols. i-iii, W.W. Fortenbaugh, P.M. Huby, R.W. Sharples, D. Gutas (Eds.), together with A.D. Barker, J.J. Keaney, D.C. Mirhady, D. Sedley and M.G. Sollenberger. Leiden 1992: Brill. (=FHS\&G).

Sullivan (1967): Sullivan, M.W., Apuleian Logic. The Nature, Sources and Influence of Apuleius's Peri Hermeneias (Studies in Logic and the Foundations of Mathematics) Ámsterdam 1967: North-Holland.

Thomas (1908): Apulei Opera Quae Supersunt, vol iii, Apulei Platonici Madaurensis De Philosophia Libri, liber PERI ERMHNEIAS, Thomas P. (ed.), pp. 176-194, Leipzig 1938: Teubner.

Van De Vyver (1929): Van de Vyver, A., "Les Étapes Du Développment Philosophique du Haut Moyen-Age", Revue Belge de Philologie et d'Histoire, viii (1929), pp. 425-452. Brussels: Société pour Le Progrés des Études Philosophiques et Historiques, 1929.

Meiser (1877-1880): Anicii Manlii Severini Boetii Commentarii in Librum Aristotelis PERI ERMHNEIAS. Prima et secunda editio. C. Meiser (Ed.), Leipzig 1877-1880. [1a abreviación usada para la primera edición es in Int; para la segunda es in Int 2,].

Wallies (1883): Alexandri in Aristotelis Analyticorum Priorum Librum I Commentarium, M. Wallies (Ed.), in Commentaria in Aristotelem Graeca, vol. 2.1, Berlin 1883. 